\title{
REPORT OF STUDIES AND THE TECHNIC FOR GOLD INLAYS
}

\author{
By ROBERT E. BLACKWELL, D.D.S., Chicago, Illinois
}

(Read before the National Dental Association, Boston, Massachusetts, August 23-27, 1920)

$\mathrm{F}$ OR convenience of description, goldinlay technic may be divided into the following parts: (1) the preparation of the cavity to receive the filling, (2) the manipulation of the wax in securing the pattern, (3) the investment of the pattern and its dissipation from the mold, (4) casting, (5) contour and finish.

The relative importance of these several steps cannot be computed upon a percentage basis; but it seems perfectly sate to say that one's failures would mount to 100 per cent unless his cavities had been properly prepared. Given a perfect cavity, it is possible to make a good inlay by any one of several methods; but no method or technic, however perfected, can compensate for an imperfection in any part of the foundation of our structure, that is the cavity.

The late Dr. G. V. Black gave to the profession a system of cavity preparation, a mastery of which, in the opinion of the writer, is essential to the highest attainments in operative dentistry. Dr. R. H. Volland ${ }^{1}$ recently emphasized this fact when he stated that we have been given a system founded upon demonstrable scientific facts, which requires only slight modifications in minor details to be adapted to the use of gold inlays. No dentist can render his best service until he has mastered the principles upon which this system is based.

${ }^{1}$ R. H. Volland, "Gold Inlays," Jour. Nat. Dental Assoc., VII (1920), 480.
Gold inlays seem to find their greatest usefulness in the bicuspid and molar teeth, particularly on the proximo-occlusal and mesio-occluso-distal cavities. Therefore this paper will be confined to the treatment of these cavities and to the construction of inlays for them. Time will not permit of a complete discussion of the principles governing the outline form of a proximo-occlusal cavity. This would involve the important subject of extension for prevention, which is too large to be taken up in detail in this paper. Altho it does not seem advisable to describe the instrumentation and technic for cavity form step by step, it does seem desirable and justifiable to emphasize at this time some points which are not being given the attention they deserve.

In a normal occlusion, the occlusal surfaces of the teeth are clean surfaces, because of the friction of mastication over them. The inclined planes of the cusps and ridges are scoured and polished during mastication so that there is no opportunity for the micro-organisms to lie in contact with tooth structure long enough for caries to occur, unless the organisms lodge in structural defects in the enamel pits or fissures. But proximal surfaces, while free from structural defects in the enamel, are not scoured by the friction of mastication over their entire area. The region gingivally of the contact point is so perfectly protected from the excursions of food that colonies 
of micro-organisms may attach themselves to the enamel and work practically undisturbed. The unclean area thus protected is called the area of liability. This area may extend buccally, lingually, and gingivally, according to the conditions. Buccally and lingually, any extension of this area will be limited by the excursions of food thru the embrasures. The contour of the proximating teeth determines the width of the embrasures; the position of the opposing cusps controls, to a large extent, the excursions of food thru the embrasures. As superficial caries does not occur under healthy gum tissue, the pcsition of the septal tissue determines, in many instances, the extension of the area of liability gingivally. It will be readily seen, then, that the factors which control the location, shape and extent of the area of liability are very variable and must be studied carefully when each case presents, for, if future caries on the surface involved is to be prevented, the entire area of liability must be included in the cavity outline and the cavity margins must be laid in areas of immunity. This usually means that the buccal and lingual margins of the proximal portion should be placed near the axial angles. ${ }^{2}$ There is no difference between the cutline requirements for gold inlays and those for gold foil or amalgam fillings. In all cases, the buccal and lingual margins of the filling should be placed where they will receive the frictional action of excursions of food during mastication. These will be selfcleansing margins which will not be the seat of future decay.

In preparing a cavity which has originated on the proximal surface it is al-

\footnotetext{
"'A strict examination of ten thousand persons applying for dental operations in the clinic of Northwestern University Dental School discovered but nine persons in whose teeth decay had spread superficially, or on the surface of the enamel, across these angles," (G. V. Black, Operative Dentistry, II, 142.)
}

most always necessary to involve a portion of the occlusal surface, that is, the marginal ridge. When this has been done it immediately becomes necessary to include more of this surface for stability and retention and, in many instances, to insure a smooth margin against which to finish the inlay (Figs. 1-3). This cutting may be executed without the least danger to the health of the pulp if one is reasonably skilful

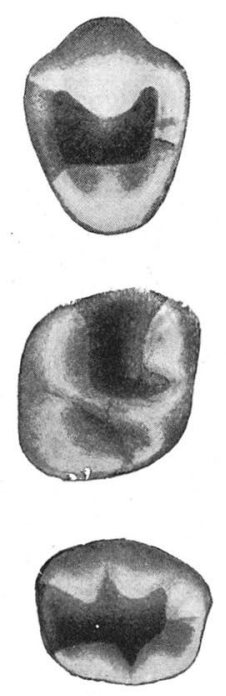

Figs. 1-3-(From Black's Operative Dentistry.)

and understands the relation of the lobes and developmental grooves to the pulp.

The resistance form of a proximoocclusal cavity is intended to provide a seat for the inlay, so that it will resist the stress of mastication. This consists of a flat seat, or seats, cut to definite angles with the surrounding walls. It is the opinion of the writer that much of the success or failure of the inlay depends upon the resistance form. Our attention has been called repeatedly to the splendid results obtained by many gold-filling operators, and it is not unusual to observe gold-foil fillings 
which have been giving excellent service for from twenty to forty years. Why? Are gold-foil fillings the only fillings which are permanent? Is the success of gold foil attributable only to its adaptability to cavity walls? Certainly gold foil, in the hands of a skilful operator working under favorable conditions, makes an excellent, durable filling; its adaptability to cavity walls is unsurpassed. But the writer is convinced that one of the most important factors in the success of gold fillings is the cavity preparation; that on account of the very nature of the material it is necessary to cut cavity walls to definite angles with flat seats. This facilitates the placing of the filling, to be sure; but its greatest lasting benefit lies in the degree of stability it gives to the filling-the stability that means permanence. If proper resistance form has increased the length of service of goldfoil fillings, in which case the most adaptable material known to our profession is used, how much more important it must be for us to study our resistance form when preparing cavities for gold inlays, where the final adaptation of filling to surrounding cavity walls is entirely by means of cement. The fact that the occlusal surfaces of inlays are carved in wax should mean the restoration of good marginal ridges. These ridges are subjected to the stress of heavy mastication by the inclined planes of opposing teeth, not now and then but repeatedly during each meal. To depend upon the flat pulpal wall alone for the resistance to this stress is not sufficient. If rocking and displacement are to be prevented and the marginal ridge is to receive the constant support it requires, the gingival wall should be flat and cut to definite angles with the buccal, lingual, and axial walls (Fig. 4).

Retention form is for the purpose of resisting tipping stress. We usually think of this form as an interlocking of the inlay with the tooth in the occlusal step; but nearly parallel walls afford frictional retention, and the resistance form, when properly provided, aids materially in preventing dislodgment. Such interlocking as is necessary can be gained readily in the deep grooves or fissures usually present on the occlusal surface, but if no imperfections are present, or if no grooves are in evidence, as may be the case in some well worn teeth, the dovetail should be cut in the position which these grooves would naturally occupy, were they present.

There has been considerable discussion from time to time about the amount of taper or inclination necessary for cavity walls as prepared for gold inlays. Dr. Ward ${ }^{3}$ had stated that this taper should be from .10 to .25 inch per inch,

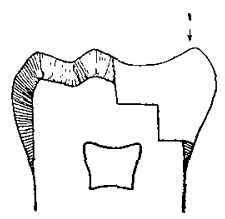

Fig. 4-Section of molar tooth cut mesio-distally in the axial plane. Arrow points to marginal ridge of inlay which should have the definite support offered by a flat gingival wall.

while other writers have usually been satisfied with a taper just sufficient to permit of the withdrawal of the wax pattern without distortion. Dr. Conzett ${ }^{4}$ has even gone so far as to prepare inlay cavities with parallel walls as for gold fillings, and claims that the cavity preparation may be identical with the preparation of gold foil, if undercuts are eliminated. It seems to the writer that the middle ground is to be preferred. Usually, if a pattern can be withdrawn without distortion and the subsequent steps can be carried out with sirict adherence to the fine detail of a satisfactory tech-

\footnotetext{
${ }^{3}$ Marcus L. Ward, "Factors of Importance in the Construction of Cast Gold Inlays," Dental Cosmos, LVII (1915), 522.

"J. V. Conzett, "The Gold Inlay," Dental Cosmos, LII (1910), 1341.
} 
nic, the inlay will be a success. But in mesio-occluso-distal cavities, particularly in molar teeth, where the distance from the axial wall of the mesial portion to the axial wall of the distal portion is considerable, and the tendency of the structure of the enamel as would be given were the cavity to be filled with gold foil. In the opinion of the writer, this practically eliminates the use of stones. Dr. Noyes ${ }^{5}$ has laid down certain rules for the preparation of enamel

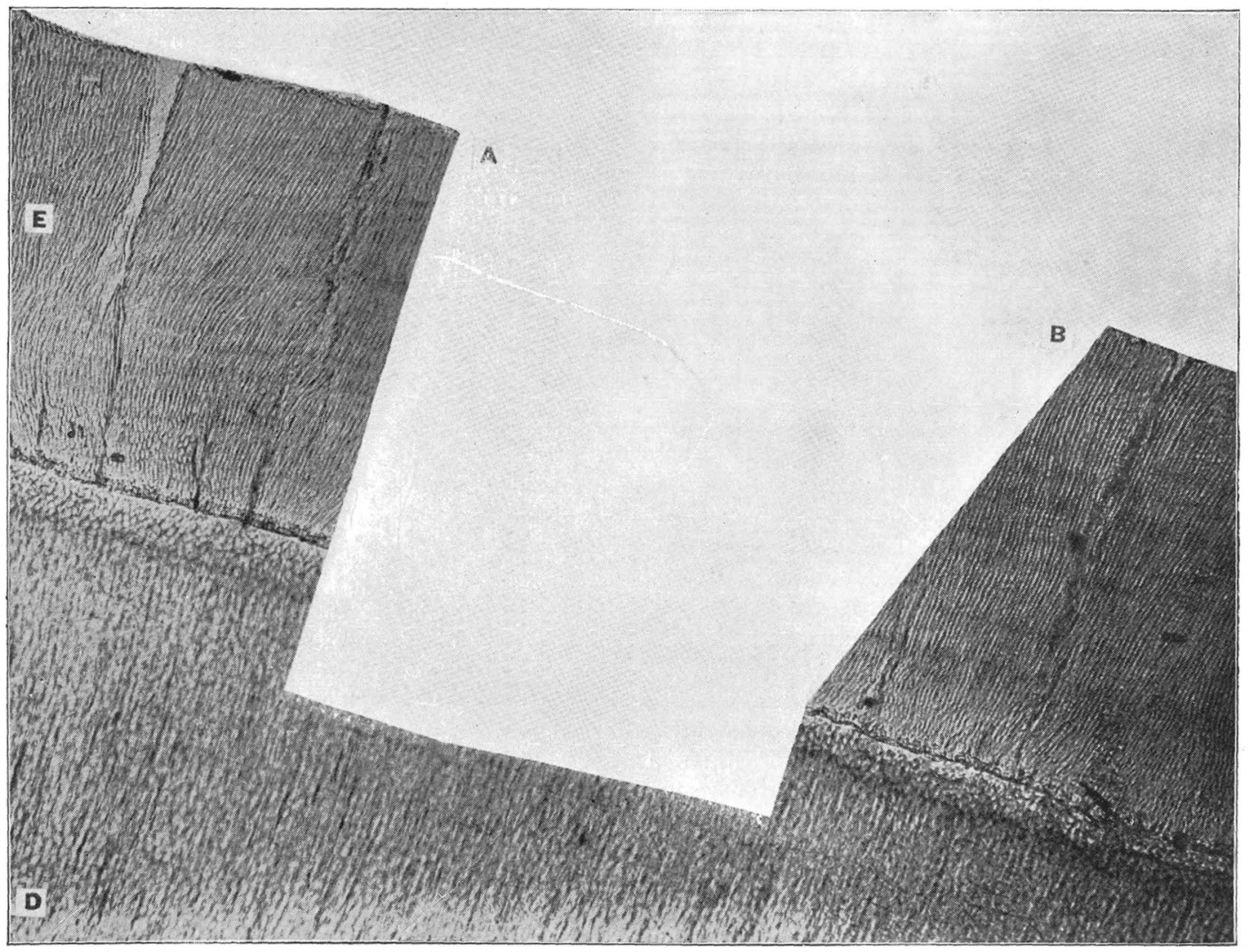

Fig. 5-A section of enamel thru which a cavity has been cut into the dentin. $E$, enamel; $D$, dentin; $B$, strong enamel wall with a strong cavo-surface angle; $A$, an enamel wall cut in such a relation to the direction , the enamel rods that it would be very frail and liable to chipping without the bevel of the cavo-surface ang shown at $A$. With the bevel shown the wall is good. (From Black's Operative Dentistry.)

pattern is to bind when removal is attempted, it seems advisable to have the axial walls well tapered in order to prevent distortion of the pattern.

The preparation of the enamel wall should be made with the utmost care, certainly with as much attention to detail and consideration of the histological walls which are applicable to cavities for all permanent fillings. These rules require that enamel shall rest upon sound dentin; the enamel rods which form the cavo-surface angle must have their in-

\footnotetext{
${ }^{5}$ F. B. Noyes, Dental Histology and Embry-
} ology, p. 80 . 
ner ends resting upon sound dentin; the rods which form the cavo-surface angle must be supported by a portion of enamel in which the inner ends of the rods rest on sound dentin and the outer ends will be covered by the filling material; the cavo-surface angle must be so trimmed that the margin will not be exposed to injury (Fig. 5). A strict application of these rules necessitates the use of hand-cutting instruments. It requires that the enamel shall be cleaved to determine enamel rod direction, which indicates chisels and hatchets; it requires that enamel walls be planed and beveled to insure marginal support, which further indicates hand-cutting instruments, including gingival margin trimmers (Fig. 6). Time will not permit of a thoro exposition of the subject in this paper; therefore, you are urged, if you have not already done so, to make an exhaustive study of the histological requirements previously referred to. It should be stated, however, that a bevel of the cavo-surface angle is not always necessary to the fulfilment of these requirements; nor is it always essential for a perfect marginal finish to an inlay (Fig. 5).

Waxes and their manipulation have most certainly received their share of discussion since the introduction of the disappearing wax pattern into inlay work. Since a good wax pattern is second only in importance to a good cavity preparation, this is not to be wondered at. From a review of the literature of this subject, it appears that there are two serious problems which must be met in pattern making. One is the everpresent problem of contraction; the other that of warpage. Some would add to these the danger of distortion upon removal; but with a properly prepared cavity and a wax which breaks before bending, when chilled to mouth temperature or lower, this danger should be elim- inated. Dr. Van Horn ${ }^{6}$ has concluded that cast pattern wax has a coefficient of expansion of about 1 per cent for every $20^{\circ}$ rise in temperature. If this be true, and it seems probable, some means must be devised to compensate for this contraction or the inlay will not fill the cavity. Dr. Van Horn's plan is to expand the pattern by re-heating; but the danger of releasing the molecular tension (elasticity) is too great, according to Dr. Prothero ${ }^{7}$ to permit of such practice. The technic which has been adopted by the section on wax patterns of the Dental Unit, ${ }^{8}$ seems to minimize the danger from the two sources which

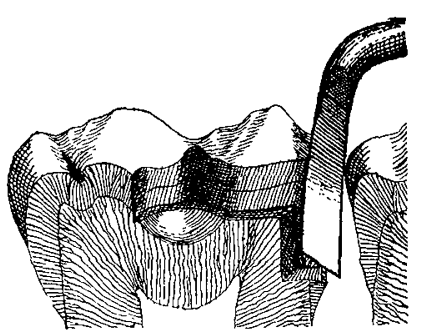

Fig. 6-The gingival margin trimmer in position giving a slight bevel to the gingival cavo-surface angle. Notice particularly the angle of the cutting edge of this instrument and its adaptation to this work. The pair of gingival margin trimmers designed for use in distal cavities serve equally well for that position. (From Black's Operative Dentistry.)

have been referred to. Unfortunately the writer is unable to give credit for this method, which is applicable to proximo-occlusal cavities. A piece of stick wax is prepared into a cone-shaped piece by passing one end quickly thru the

${ }^{\circ} \mathrm{C}$. S. Van Horn, "The Importance of Recognizing Temperature Changes and the Sequelae When Executing Cast Gold Inlay Technique," Dental Items of Interest, XLII (1920), 371.

${ }^{7}$ J. H. Prothero, Prosthetic Dentistry, p. 948.

${ }^{8}$ The term as here used, and elsewhere in this paper, refers to the G. V. Black Dental Unit of Northwestern University. 
Bunsen flame and then, while holding the stick in one hand, pushing back the softened exterior with the thumb and finger of the other hand. This is done again and again until the cone of wax is soft on the outside but is hard and firm on the inside or core. The tip of the cone will be soft thruout. The hardened core acts as a plunger to drive the softened wax into the angles and against the margins of the cavity. When the cone is prepared, the excess wax is broken off and the point of the cone pressed into the gingival portion of the cavity. Pressure in one direction is continued until the entire cavity has been filled and the tips of the cusps are felt thru the wax by the thumb or finger for, say, a minute. The wax is then chilled with room temperature water. With a sharp instrument the excess wax is quickly cut away from beneath the bell-crowned portion of the tooth and the mass is lifted from the cavity with the thumb and finger, the bulk of wax which bulges out to the buccal and lingual being used as a handle. All margins are well protected at this time by excess wax. With a small sharp knife the proximal portion is cut to form, and the gingival margin trimmed so as to leave a very slight excess, which subsequently should be burnished against the gingival margin of the cavity. The wax is reinserted into the cavity and the pattern trimmed to form with suitable instruments, which should be cold. After all margins are burnished, the pattern should be chilled again with room temperature water and removed by means of a fine exploring tine. The pattern may now be immersed in a bath of room temperature water and invested when convenient. There are four items in the technic just briefly described which need some emphasis. First, the cone of wax must be so shaped that the tip will be pressed against the gingival wall first (Fig. 7) in order that as pressure is applied the wax may be condensed against the gingival wall. This should help to obviate the gingival shrinkage of which some operators complain. Second, the continued pressure upon the wax, as it cools to mouth temperature, tends to keep the cavity full and also condenses the wax. Third, the manipulation of the wax with cold instruments, trimming and burnishing, adapts the pattern to the cavity margins and aids in compensating for possible loss in volume by shrinkage. Fourth, chilling the wax with room temperature water and the maintenance of that temperature lock the wax in its new form and prevent distortion, which might take place

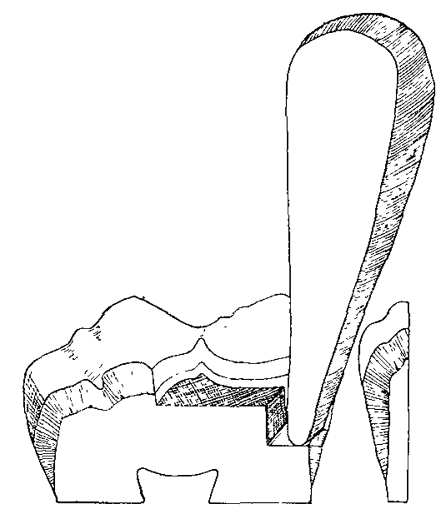

Fig. 7

were the temperature raised and the elasticity of the wax released. For mesiooccluso-distal cavities this technic is not so well adapted, and consequently a different technic is advised. The cavity must be prepared so that the crown of the tooth will not be any wider mesiodistally at or near the occlusal than it is at the level of the gingival walls; that is, all of the bell crown must be cut away. An adaptable matrix must be adjusted to fit the tooth, but not too tightly. The matrix is filled with wax somewhat softer than is commonly used; then the wax and matrix are forced over the tooth and cavity and pressure is applied over the whole mass with thumb or finger. This is held a moment or two, chilled with room temperature water, and 
matrix and wax removed. The cavity surfaces of the wax are examined to determine whether the cavity has been copied accurately. If so, the matrix is removed and the pattern carved and trimmed as in the technic described for proximo-occlusal cavities. In case the cavity has not been accurately copied in the wax, it is best to make a new start with freshly softened wax.

The sprue former should be placed in such a position as to permit the gold, when cast, to flow readily to all parts of the mold with equal force. That is, in proximo-occlusal cavities, it should be placed so as to bisect the angle formed by the junction of the proximal portion with the occlusal portion of the wax; in mesio-occluso-distal cavities, it should be placed at, or near, the center of the occlusal portion. It is a good plan to remove the pattern from the cavity upon an exploring tine, place it in the hollow of the hand, heat the sprue former in the Bunsen flame while holding the metal in the naked fingers to prevent overheating, and to touch it to the pattern quickly and at the same time direct a mild blast of air upon the wax so that the sprue former is immediately affixed. The pattern should now be cleansed preparatory to investing. Washing the pattern with alcohol or soap water will remove mucus or oil, but temperature changes due to evaporation of these liquids from the pattern should be considered. The section on investments of the Dental Unit reports that the evaporation of alcohol from a wax-covered thermometer bulb caused a lowering in temperature of $13^{\circ}$ after the expiration of thirty seconds and of $16^{\circ}$ after sixty seconds. The same wax-covered bulb, washed with room temperature water, caused a change of $5^{\circ}$ after thirty seconds and $8^{\circ}$ after sixty seconds. These changes are not great but should be reckoned with if one desires to follow accurately the room temperature method. To obviate these changes due to evaporation of liquidcleansing agencies, the pattern should be bathed in an excess of liquid-especially if alcohol is used-and then quickly immersed in a bath of room temperature water and left there until the investment material is mixed and ready for immediate application.

In order to insure an investment of uniform consistency, it is advisable to measure the investment material and water for each mix. They will also result in a saving of material. Both water and investment material should be used at room temperature. This uniform temperature can easily be had at all times if a bottle of water and a can of investment material are always allowed to stand side by side on the laboratory bench. Most investments in which silex and plaster of Paris are the principal ingredients should be mixed to a thick creamy consistency. The pattern should be covered with a thin film of material, using a sable's-hair brush as an applicator. The investment is painted on the pattern with a brushing motion toward the marginsnot a stippling motion-in order to avoid the lodgment of air bubbles on the pattern or the entrapment of air on the cavity side of the wax. More material is applied until the entire pattern is thickly covered; then the ring is placed in position on the base and the investment poured into it until the ring is full. The ring should not be tapped while the investment is being made, as that may cause air particles to unite and form bubbles, some of which wili settle on the cavity side of the pattern and result in nodules of gold on the inlay. After the expiration of fifteen or twenty minutes, the base and sprue former may be removed and dissipation of the wax should begin.

The object of heating the invested pattern is to remove the wax and leave a mold into which to cast molten gold. But the effect of heat upon the investment must be considered. Dr. Prothero ${ }^{9}$ has stated that the binder in investment

${ }^{9}$ J. H. Prothero, op. cit., p. 950. 
materials is usually plaster of Paris, which "decomposes to a more or less extent at $190^{\circ} \mathrm{C}$." or $372^{\circ} \mathrm{F}$. Naturally this would vary with the quality of plaster used. Accepting this figure as approximately correct, it seems advisable to dissipate the wax at a temperature between $320^{\circ}$ and $350^{\circ} \mathrm{F}$. That this can be done has been adequately demonstrated. Several devices have been suggested to regulate the temperature during dissipation of wax. ${ }^{10}$ Gas and electrically heated ovens have been used with equally good results. The section on investments of the Dental Unit has adapted a gas thermostat to an oven made of galvanized iron which can be regulated to maintain the heat at practically any temperature desired. Some dentists have used old vulcanizers. But a very satisfactory result can be obtained by placing the flask on a metal plate over the Bunsen flame. The flasks should be raised slightly from the plate to permit of the free circulation of air under them-a piece of wire screening will answer-and they should be at least partially surrounded by a metal shield as a protection against drafts. A few thermometer tests will determine how much gas should be used, and the gas cock can be opened to the same position each time. ${ }^{11}$ The flasks should be placed in the oven, open end down, so that the wax can run out as it melts, and the temperature kept at $340^{\circ} \mathrm{F}$. for an hour to an hour and a half, according to the size of the pattern. They may then be removed and before casting should be allowed to cool to room temperature. The advantages of this method of wax dissipation are these: The wax is not

\footnotetext{
${ }^{10}$ R. H. Volland, "Casting Gold Inlays," Jour. Nat. Dental Assoc., VI (1919), 425.

${ }^{11}$ The bulb of an ordinary vulcanizer thermometer may be invested in an inlay ring with the same investment material which is being used for inlays. This should be kept on the plate with the flasks and used as a guide to control the temperature until the amount of gas required to produce a temperature of $340^{\circ} \mathrm{F}$. has been determined.
}

boiled out of the mold, with consequent injury to the surfaces of the mold by explosions of gas; the strength of the investment is not impaired by overheating; the mold is not distorted by excessive expansion of the investment; and the method is simple. Of the points mentioned, the strength of the investment is probably the most important. Dr. Prothero $^{12}$ states that "decomposition of the binder from overheating is largely responsible for the roughening and bulging outward of the matrix walls under the pressure of casting." Some writers, among them Dr. Lane, ${ }^{13}$ have advocated rounded cavity angles because of the danger of the molten gold rounding sharp angles in the mold. The writer maintains that if the binder has not been overheated no angle is too sharp to be reproduced in the casting. The reproduction in the inlay of the very small hole made by the exploring tine used to remove the pattern from the cavity, has many times demonstrated the strength of the investment material after dissipation of the wax by this method.

Of the various devices suggested for casting inlays, none is without merit; but for casting molten, not superheated, gold into a mold which has been allowed to cool to room temperature a centrifugal machine should be chosen, since it will allow room temperature to be maintained in the mold and will cast the inlay quickly enough to reproduce accurately sharp angles. With such a machine there is no gas, air, nor steam pressure to cool the gold; it can be heated just until it spheroids and the surface is clear; then the cast can be made. It should not be heated longer or to a higher temperature. Longer heating will raise the temperature of the flask much above that of the room and will cause expansion of the mold, which in itself might not be undesirable were it not for the ever-present danger of

\footnotetext{
${ }^{12}$ Op. cit., p. 950.

${ }^{18}$ J. C. Lane, "The Casting Process Applied to Gold Inlays," Dental Digest, XV (1909),
} 369. 
distortion, which goes hand in hand with expansion. Superheated gold is the cause of many rough castings. Most of the centrifugal machines on the market today provide for the melting of gold in a crucible outside the flask. This is a very desirable feature. The casting section of the Dental Unit has given the writer the results of some very interestirg experiments which were conducted to show the temperature of the mold at the time of casting. In all cases tried, the mold was at room temperature when fusing of the gold was begun and temperature readings were made as soon as the ingot (3r/2 dwt. of $22 \mathrm{~K}$ gold) was in a condition to be seconds, and the temperature of the flask was never increased more than $2^{\circ} \mathrm{F}$. when the Volland centrifugal machine was used.

When the inlay is placed in the cavity, there will not be contact with the proximating tooth, as the wax contact was cut away while the model was being trimmed. Therefore the inlay may be forced into place immediately without much trimming, and it may be determined at once whether or not it is a success. As the higher carats of gold are quite soft it is necessary that the contact point shall be restored with solder to insure a permanently well-rounded contact point, one

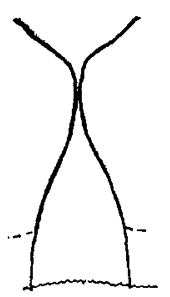

A

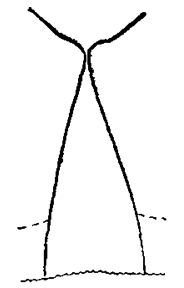

B

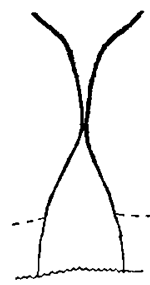

C

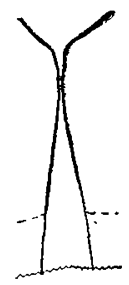

D

Fig. 8-Good and bad forms of proximal contacts, buccal view. The proper position of the contact point bicuspid and molar teeth is about as shown in $A$, just a little to the gingival of the marginal ridges. The convexity of both surfaces at this point should generally change to a slight concavity in the gingival direction. The position of the contact in $B$ is too far occlusally. With such a form of contact, less than the normal portion of food will pass thru the embrasures, and the exposed portions of the proximal surfaces of the teeth will not be normally cleansed in mastication. The position of the contact in $C$ is too far gingivally. The long approaches from the occlusal invite the wedging of food between the teeth, with the danger of forcing the contact open. The position of the contact in $D$ is about right, but the teeth have not been separated to restore the normal mesio-distal width of the interproximal space. (From Black's Operative Dentistry. )

cast. The experiments were not exhaustive, but were sufficient to show that if one desires to cast gold into a mold exactly at room temperature he should use a centrifugal machine with a shallow crucible set apart from the flask and a flame much hotter than any which a gas and air blowpipe will produce. With an oxygen blowpipe the ingot was fused and ready to cast in fifteen to twenty that will not be flattened by interproximal wear.

The form of the contact point should be smoothly rounded, and it should be placed in its normal position, a little to the gingival of the crest of the marginal ridge. Dr. Black has stated that the contact point should be as two marbles touching each other. This means that there should be a rounding away of the 
proximal surface of the inlay in all directions from the point of contact. A great many contact points are placed so far to the occlusal that the margins of the filling do not receive the natural cleaning. For when the contact point is placed, say in a millimeter gingivally of the crest of the marginal ridge, the proximating marginal ridges are thrown farther apart (Fig. 8A), thus forming a larger opening between the inclined plans of the ridges, which will accommodate a correspondingly large amount of food. The food is separated by the contact point and forced thru the buccal and lingual embrasures by the cusps of the opposing teeth. Also the area not

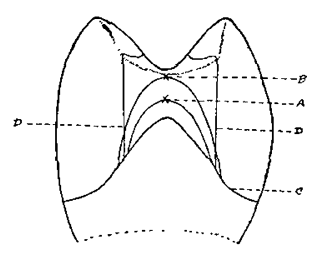

Fig. 9-Proximal aspect of an upper bicuspid. $A$ indicates correct position of contact point, which is the same as $A$, Fig. $8 ; B$ indicates contact point too close to the marginal ridge as in $B$, Fig. $8 ; C$ indicates the position of the septal tissue. $D$ indicates probable position of filling margins. Note the difference in size of the unclean areas as indicated by the curved lines running bucco-gingivally and linguo-gingivally from the contact points $A$ and $B$. It will be seen that the filling margins are safer when the contact point is placed somewhat gingivally of the crest of the marginal ridge.

scoured by food will be made more narrow bucco-lingually and the margins of the filling will be more certain of being kept clean by natural means, if the contact point is in its normal position (Fig. 9 ). When the contact has been adjusted to proper form and tightness (Fig. 10), all the margins should be thoroly burnished by heavy hand pressure with suitable instruments before setting. After cementation, the margins should be fin- ished with sandpaper disks and strips; but burnishing should not be resorted to on account of the likelihood of fracturing the hardened cement.

The value of the gold inlay as a means of restoring lost contour has been much discussed. Some men consider its utility in the restoration of occlusal surfaces its greatest worth. Certainly this is of great value; but the writer cannot agree with those men who believe that unless every line and wrinkle of the normal occlusal surface is reproduced in the filling, masticatory function is

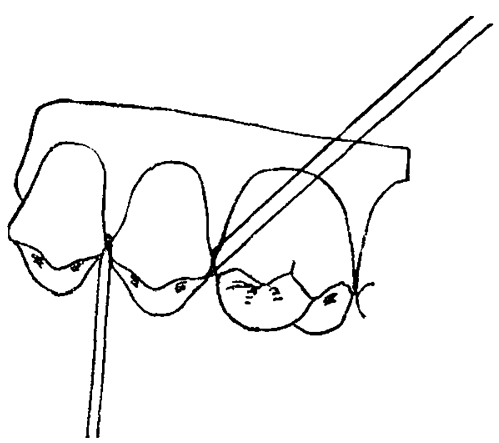

Fig. 10-In testing a contact the ligature should be first carried thru from occlusal to gingival, then the two ends of the ligature should be held paraliel in the occlusal direction, as shown between the two bicuspids; the distance between the strands will indicate the bucco-lingual width of the contact. The two ends should then be held paralleI in the buccal direction, as shown tetween the second bicuspid and first molar; the distance between the strands will indicate the occlusogingival width, of the contact. In either position, if the parallel strands are more than from $1 \frac{1}{2}$ to $2 \mathrm{~mm}$. apart, the contact is too broad. (From Black's Operative Dentistry.)

impaired. A proximo-occlusal filling, however, is nothing better than a plug for a carious cavity, unless full proximal contour with strong contact is restored. The fact that the gold inlay offers the easiest, surest and best means of restoring lost contour gives it a position in the first rank as a filling for cavities in these positions where there is danger 
of injury to the gingivae because of matter of contact (Fig. 11). Or the impaction of food. Not only is it a lack of contact of one filling may have simple matter to restore the contact between two surfaces, but frequently it is possible and practicable to restore several contacts on one side of the arch by a little over-contour of one inlay which may be driven into position subsequent caused scveral teeth on one side to move apart just enough to allow food to crowd in thru the weakened contact points and press down upon the gingivae, causing annoyance to the patient and injury to the septal tissue. Such conditions, seri-
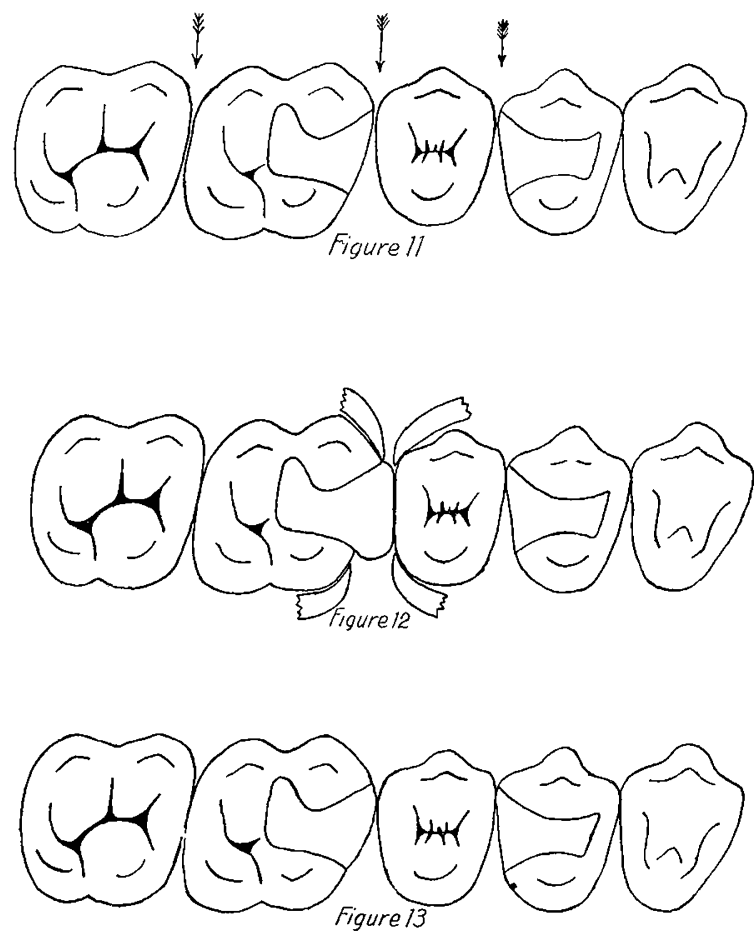

Figs. 11-13-Fig. 11, occlusal view of the upper right cuspid, first and second bicuspids and first and second molars. Arrows point to lack of contact or weak contact. Fillings in first molar and second bicuspid. Fig. 12, the jaws of the Perry separator are lifting the first molar and second bicuspid apart sufficiently to tighten the contacts between the first and second molars and the first and second bicuspids. Gutta-percha is shown packed into the first molar cavity after the removal of the old filling. Fig. 13, an inlay, somewhat over contoured, has been placed in the molar cavity. It holds the separation previously gained and restores three faulty contacts to proper tightness.

to the movement of the teeth by slow separation.

It not infrequently happens that a patient presents with several fillings in the same region which may have been well done except for the very important ously threatening the health of the peridental membrane, may be greatly improved in many instances by the placing of one inlay. The Perry separator should be adjusted in one or more positions to determine which contacts can be 
tightened (Fig. 12). Usually a filling can be removed and the cavity packed with base plate gutta-percha to hold the separation gained with the mechanical separator. If the teeth need to be moved a considerable distance, the patient may return several times and additional separation may be gained with each sitting. Finallv, an inlay mav be made which must be over-contoured somewhat and driven into position. This will tighten the contacts and frequently will remedy the cause of the gingivitis (Fig. 13).

To be sure, contacts may be tightened in a similar manner with gold foil or amalgam fillings, but the gold inlay seems to lend itself more readily to such operations. Occasionally, when teeth are moved in this manner, it will be necessary to grind one or more cusps a little, so that the contacts in the opposing arch will not be disturbed even by the slight change in conditions.

An attempt has been made to outline a technic for the construction of cast gold inlays which has proved in the hands of the writer and in the hands of others with whom he has been associated, good and workable. The results obtained have been superior to those obtained by any other method. It is not claimed that this is the one and only method, nor that inlays made after the manner described will be perfect every time a cast is made; but is is claimed that this reasonable, simple, and yet scientific method may be developed into a technic which will bring satisfactory results in a very large percentage of cases.

\section{DIscUSSION}

Daniel H. Squire, Buffalo, New York: The longer we are engaged in dental practice the more we are impressed with the fact that many operations which are equally successful are made by as many different plans of procedure; yet the basic principles which this paper presents should be the foundation of these various methods. The age of the patient, the amount of decay present, the condition of the teeth relative to fractures, the reaction of the saliva, the tendency toward inflammation of the supporting structures, to- gether with the general hygiene of the mouth, and the home care which the teeth receive are factors which will influence the outline, as well as the resistance form of the classes of cavities. In order to make the inlay safe against future dislodgment, and recurrent decay, cavities in general should always be made broad enough and deep enough to hold a sufficient amount of filling material to resist the stress of mastication; that is, the metal should be of sufficient bulk to be strong in itself, otherwise fracture will occur at the junction of the occlusal and the proximal portions.

The cavity formation which is advocated by the essayist both in regard to the shaping and the finishing of the dentin and enamel walls is most important, because the unseating of inlays and the fracture of enamel walls may be traced to narrow seats, indefinite angles, extreme divergence of the dentin walls, and to enamel margins with rods unsupported by dentin. Fractional resistance is a very important item, one which adds much toward the stability of the inlay, as it is retention in itself to quite a degree. Experience compels me to say that if these classes of cavities are prepared under the influence of anesthetics a review of dental anatomy in respect to the location of the pulp and of pathology in regard to the etiology of hyperemia and its possible results would not come amiss. The contraction of the wax pattern has been one of the principal features in the failure of inlay work, and the method of using a cone of wax will obtain a more perfect adaptation, as it is founded upon the same principle which is employed in the use of modeling compound in impression taking by the indirect method. Not all of the contraction of the material, however, takes place at the gingival seat, because it is equally apparent at the extremity of the occlusal portion of the cavity. I have used a full band copper matrix and found it of great assistance in confining the wax while it is being forced to place. The band is applicable to any tooth without separation, as it may be reduced in breadth to accommodate itself to the occlusal side of the contact point of the tooth on the one side and allowed to project just beneath the wall of the cavity on the other. It is also loose enough to permit the wax to pass over the gingival margin of the cavity, thus assuring a perfect adaptation. The wax and matrix are removed without danger of distortion. The narrow portion of the band is clipped with scissors and easily separated from the wax pattern after the wax has been trimmed, replaced in the tooth, and the occlusion practically determined. I believe it was Dr. Schayes who advocated the softening of the occlusal surface of the wax with a sufficiently warm burnisher so that 
the patient could reproduce the relation of the inclined planes of the cusps upon the wax by oscillating movement of the jaws. This will give a perfect occlusion without changing the pattern in any way, because only the surface of the wax is slightly softened and the final burnishing may be done with cold instruments.

The same principles which are advocated by Dr. Blackwell relative to the shaping of the wax cone and its adaptation to proximoocclusal cavities are also applicable to the mesio-disto-occlusal. It has been my practice to use two wax cones of suitable size, fastened together in a perpendicular relation, and with a full band matrix already fitted and adjusted about the tooth. The cones are warmed and forced quickly to place, the apex of each cone coming in contact with the gingival seat of the corresponding side. The wax is held in position until cooled and then removed. While I do not dispute the claims of the essayist for the merit of the large cone, it is my opinion that greater accuracy may be obtained with the use of two small ones. The essayist does not mention any particular investment material, but in order to bring the discussion to some specific point I may be permitted to refer to the Taggart investment, as it is the one with which I am more familiar. After this investment has been thoroly mixed, a small amount is poured upon a glass slab and the thin, watery portion transferred to a piece of paper, for the paper will absorb enough of the moisture to leave the investment slightly thickened and it can then be painted upon the wax without danger of its being disturbed when the rest of the investment is poured into the ring. There is less danger of entrapment of air when this method is employed. In the heating of this investment, a very low temperature is maintained for fifteen or twenty minutes, or until no steam or gas appears to be rising from the mold, and then it is subjected to a higher temperature until the investment is quite near the inner side of the ring. It is then removed from the fire and allowed to cool at room temperature.

My experience has been with the direct pressure machine of the Taggart type, and I am of the opinion that the investment need not be excessively heated during the process of casting if the gold is placed upon a charcoal block and brought to a high degree of temperature; it should then be transferred to the ring and the melting of the gold finished with an oxygen blowpipe. However, I am very much impressed with the technic which Dr. Blackwell has suggested for this part of the work.

The opportunity which is offered for closing open contacts, when restoring teeth to their normal forms is one of the most important items which has been mentioned in this essay. The investigations of Dr. Black and the articles which he has published regarding the contact of teeth show beyond a doubt that a lack of contact, or improperly placed contact points, are the very beginnings of disease of the septal tissues. The proper placing of the contact point is extremely important, as well as the proper contour of the proximal surfaces of the teeth to be restored. There are many large inlays made with very prominent contact points, but with such slab-sided proximal surfaces that the food is forced into the interproximal spaces just as freely as if no contact had been made. Inlay work is not a short cut over the malleted gold filling by reason of its technic, but on the other hand a most painstaking process of restoration.

Earle S. Smith, Iowa City, Iowa: For many years the dental profession prepared cavities for gold foil and for amalgam fillings in a variety of ways-one man made them one way, another man made them a different way-and it took a Dr. Black to standardize the technic and to present the scientific principles upon which all work of cavity preparation should be based. These principles have been largely accepted by the profession.

The history of the making of gold inlays has been very similar to the history of cavity preparation-one man has been making them one way, another in a different way-and as a consequence uniform results have not been obtained. On account of the careless, slipshod methods of some men, the gold inlay has received much unjust criticism. It has been the abuse and not the use of the gold inlay that has caused much of this criticism. If it were essential that there be a standardized technic based upon scientific principles for the preparation of cavities, to a like degree is it essential that a standardized technic for the making of gold inlays be adopted.

As has been suggested by the essayist with a few modifications the principles of cavity preparation, as laid down by Dr. G. V. Black, should be used as the basis for preparing cavities for inlays. One step not mentioned by the essayist but which should be done first of all is the examination of the occlusion. The making of check bites with wax and even in some cases the making of casts will often disclose points whereby the inlay may be curved to bring out the greatest amount of efficiency in the finished inlay.

The biggest factor in the retention of an inlay is the resistance form of the cavity. Even the dovetail made in the occlusal part of the cavity in the strictest sense is resistance form and not retention form; it is made to prevent the inlay from being displaced proximally and that displacement would be due to a force 
acting parallel to the long axis of the tooth and toward the pulpal wall or toward the gingival wall. Many inlays have been failures because the gingival wall of the cavity has been concave instead of flat. Undoubtedly many of you in your youthful days out on the old farm used an old-fashioned wash basin. Some mornings after a good cold night you found that water had been left in the basin the night before and it was frozen solid. How did you remove it? You set the pan on the stove for a few moments then pushed downward upon one edge of the ice and the piece slid right out of the pan. Why? The resistance form of the pan was concave. Neither must the cement be relied upon to retain an inlay. An inlay cemented to place with an inferior cement but in a cavity having good resistance form has a much better chance to be a success than an inlay cemented to place with the best cement but in a cavity with a poor resistance form. It is absolutely essential that the walls be smooth, that the gingival and pulpal walls be fiat, and that the other walls meet the pulpal and gingival at but slightly obtuse angles.

Dr. Blackwell has given some good suggestions about the manipulation of wax. In making form pressure must be maintained until the wax has cooled; otherwise the elasticity in the wax will cause it to draw away from the gingival wall. Another method for the manipulation of wax for mesio-occlusodistal cavities is to soften the wax similar to the way suggested by the essayist, pinch the softened end of the wax to a wedge form and then with a knife split the wedge so as to form two small points. After a slight reheating the wax can be introduced into the cavity, one point in the mesial portion, one in the distal, and a generous supply into the occlusal and the wax forced "home." For carving proceed the same as for a simple proximal. This does away with the necessity of using a matrix.

I do not agree with the essayist about leaving the wax form in water for any length of time. The sooner it is invested the least chance there is for distortion or for accidental breakage.

The things desired in a mold are that it be the same in size as was the wax pattern, that it have smooth walls, and that these walls be sufficiently strong to withstand the impact of the molten gold without being distorted. The dissipation of the wax is the point where many operators have failed. Excessive heat has spoiled many an inlay because the binder of the investment has been abused, leaving a mold with pitted walls and different in size from the wax pattern. A low heat, $370^{\circ} \mathrm{F}$. or below for a long time is better than a high excessive heat for a short time.

By leaving the sprue hole down when the investment is on the fire, one takes advantage of all of the three ways that wax is dissipated-volatilization, melting and being absorbed by the investment, melting and running out of the sprue hole.

If a low degree of heat is desirable while the wax is being dissipated, then for the same reason the investment should not be subjected to high heat when the gold is being melted. casting should be done in a cold mold and with gold that has not been superheated.

A standardized technic if it is to be adopted by the profession as a whole and if it is to accomplish the most good must be simple and yet accurate and efficient. Dr. Blackwell has outlined a technic that requires no speciai high-priced equipment; it is a technic that no person with the average amount of skill will have difficulty in following and it gives accurate results; it is a technic that fulfils all of the requirements-simplicity, efficiency, accuracy.

Dr. Blackwell: I am glad that the discussers have given emphasis to some points. I should like to suggest that if any of you have not made a number of proximo-occlusal or mesio occluso-distal inlays for technic cavities you should make them. That is the only sure way to check up on your work. In the mouth, you do not always see every imperfection, especially when there is a slight deficiency at the gingival margin; but you do see them in the technic cases, and by continually working and improving your technic such imperfections can be overcome. I believe that all of us should do a great deal of this sort of work if we are to render our best service to our patients. 